\title{
1 TITLE: Snail host parental investment throughout a Schistosoma mansoni infection
}

2 AUTHORS: Stephanie O. Gutierrez ${ }^{1}$, Olivia J. Lockyear ${ }^{1}$, Dennis J. Minchella ${ }^{1}$

${ }^{1}$ Purdue University, Department of Biological Sciences, West Lafayette, Indiana

Correspondence should be sent to Stephanie Gutierrez at: gutier68@purdue.edu

Abstract: Parental investment theory describes the ability of organisms to respond to an environmental challenge by increasing the fitness of future offspring. Utilizing life history

8 changes, organisms can maximize fitness by increasing their total reproductive output or by

9 investing more into the success of fewer offspring. In cases where parasitic infections result in

10 castration of their host, increased reproductive effort known as fecundity compensation has been

11 demonstrated in a variety of organisms. This response appears predictive of expected future

12 reproductive losses. Organisms struggling with an environmental pathogen, may attempt to

13 better prepare their offspring for the environment they are experiencing through

14 transgenerational immune priming (TGIP). In immune priming, primary infection lowers the

15 prevalence and intensity of a subsequent infection by the same pathogen. Transgenerational

16 immune priming carries pathogen resistance into further generations without genotypic changes.

17 The focus of this study was to determine whether invertebrate parental investment into offspring

18 parasite resistance varies over the course of an infection. Utilizing the snail host Biomphalaria

19 glabrata and its trematode parasite Schistosoma mansoni, offspring were reared from specific

20 time intervals in the parent's infection and subsequently exposed to the same pathogen when

21 each cohort reached the same age- 12 weeks. Differences in infection prevalence and intensity

22 were expected based on when the offspring were born during their parent's infection. A trade-off

23 was predicted between the number of offspring produced in a cohort and offspring resistance to 
24 future infections. Offspring born during the period of fecundity compensation were predicted to

25 exhibit lower resistance due to a dilution of individual investment by parents into a larger

26 offspring pool. While our results did not support TGIP, there were differences in offspring

27 prevalence, as well as an indication that parent health may interact with genetics in offspring

28 resistance. Results suggest that parental condition can influence resistance of $B$. glabrata

29 offspring to $S$. mansoni but that TGIP may not be operating in this system.

30

31 Keywords: Parental investment; Schistosoma mansoni; Biomphalaria glabrata;

32 Transgenerational immune priming; Fecundity compensation.

33

34

35

36

37

38

39

40

41

42

43

44

45 
46 Resource limitations as well as environmental burdens can impact an organism's ability to

47 maximize reproduction and result in trade-offs between life history traits. For instance, Daphnia

48 magna infected with Pasteuria ramosa can trade infection tolerance for an ability to maximize

49 reproductive output (Vale and Little 2012). This life history response is termed fecundity

50 compensation, as exposed hosts have a burst of early reproduction to compensate for the

51 expected future loss of reproductive success (Minchella and Loverde 1981). Fecundity

52 compensation can be seen in cases where parasites ultimately end a host's ability to reproduce

53 once infected, as hosts must choose between maximizing their defense against the parasite or

54 maximizing their reproduction prior to castration by these parasitic castrators.

Schistosoma mansoni is a parasitic castrator in its intermediate snail host and a disease agent in humans. Schistosomiasis, a parasitic disease caused by Schistosoma spp., afflicts more

57 than 250 million people worldwide and remains endemic in parts of Africa, Asia, and South

58 America (Sokolow et al. 2016). Humans are infected by free-swimming cercariae released by

59 infected snails in water sources. Subsequently, the parasite is recirculated into snail populations

60 by infected human waste matter reaching freshwater sources, where miracidia can hatch and

61 infect Biomphalaria spp. snails. Understanding snail host life history responses to parasites can

62 provide a better understanding of host-parasite relationships.

63 Organisms struggling with an environmental pathogen, may attempt to better prepare

64 their offspring for the environment they are experiencing. Immune priming occurs when a

65 primary infection lowers the prevalence and intensity of a subsequent infection by the same

66 pathogen. The occurrence of immune priming in invertebrates challenges a long-standing

67 assumption that invertebrate immunity is non-adaptive and without memory. In the B. glabrata

68 and S. mansoni system, snails vaccinated with miracidium protein extracts prior to parasite 
exposure exhibited a lower prevalence of infection and lower infection intensity (Portela et al.

70 2013). Furthermore, the innate immune memory in B. glabrata has been determined to be a

71 humoral immune defense response rather than one based in cellular mechanisms (Pinaud et al.

72 2016). This within generation immune priming is widespread in invertebrates, and there is

73 growing evidence that invertebrate immune priming can be transgenerational (Roth et al. 2018).

74 Transgenerational effects characterize the altered offspring phenotypes generated by

75 parents exposed to an environmental stimulus. Transgenerational immune priming (TGIP)

76 thereby carries changes in immunological function across generations without genotypic

77 changes. For example, in Daphnia magna, maternal and offspring environments were both found

78 to be major determinants of disease susceptibility. Parental investment into offspring immunity

79 differed depending on the mother's developmental environment, as offspring from stressed

80 mothers were more resistant than offspring from unstressed mothers (Mitchell and Read 2005).

81 While TGIP has been well documented in vertebrates with the maternal transfer of immune

82 factors which reduce offspring susceptibility to disease, research of TGIP in invertebrates has

83 been more limited (Littlefair, Laughton, and Knell 2017).

84 The focus of this study is to determine whether a snail's parental investment into

85 offspring immunity changes over the course of a $S$. mansoni infection. In the infected generation

86 of parent snails, we expect to observe fecundity compensation prior to parasitic castration.

87 Rearing the offspring produced at different times following the parent's parasite exposure, our

88 objective is to characterize the resistance of offspring by challenging them with the same

89 pathogen when they reach the same age. Offspring resistance was measured as infection

90 prevalence, while parasite virulence was estimated by cercarial output (the parasite's

91 reproductive success) (Nowak and May 1994). We hypothesize that there will be evidence of 
92 TGIP, which would be revealed by a lower infection prevalence and/or a lower infection

93 intensity in offspring from an infected parent. Furthermore, we predict that there will be

94 differential resistance in offspring depending on the time at which those offspring were produced

95 in their parent's infection. We expect offspring laid early in a parent's infection (before fecundity

96 compensation and subsequent parasite development) will demonstrate the highest resistance,

97 evidenced by a low prevalence and virulence, due to a parent's ability to allocate more resources

98 to offspring before the host life history response and then the infection take their toll.

The NMRI strain of S. mansoni was maintained in our laboratory utilizing B. glabrata

100 snails and Balb/c mice. All snails were grown under controlled laboratory conditions with a

101 twelve-hour light and dark cycle and a room temperature of $25^{\circ} \mathrm{C}$. Snails were housed in jars of

102 well water and provided fresh lettuce ab libitum for the duration of the experiments.

A total of 198 snails between 9-15mm in length were initially divided into an

104 experimental parent group of 150, and a control parent group of 48. Snails from the experimental

105 group were individually exposed to five miracidia, and the control group snails were sham

106 exposed in well plates, where they remained for twelve-hours before being transferred to

107 individual jars of $225 \mathrm{~mL}$ volume. Styrofoam was placed in each jar as an egg-laying substrate.

108 Egg masses were collected from the Styrofoam and separated out for ten weeks at discrete

109 weekly intervals (Figure 1). Fecundity was recorded weekly, taken as the number of egg masses

110 laid per individual each week (Gleichsner, Cleveland, and Minchella 2016). Egg collection

111 coincided with weekly water changes, as parents were moved to new jars of well water. After

112 five weeks, non-laying and dead parents were removed from the experiment, culling 19 parents

113 from the experimental group and 8 parents from the control group. Parental infection was

114 verified by the release of cercariae in the remaining experimental group 5-6 weeks following 
115 parasite exposure. Snails were placed in well-plates and exposed to a light for 1 hour to stimulate

116 cercariae emergence (Lewis et al. 1986). The proportion of experimental parents infected

$117(n=131)$ was recorded as a measure of prevalence. Uninfected snails in the experimental group

118 were removed and the control group was randomly culled to 25 individuals. Offspring collected

119 from removed parents were discarded from the experiment.

120 Offspring were differentiated by both their parent ID and week of collection following

121 the parent's parasite exposure, which was designated post exposure (PE). Offspring from both

122 control and infected parents were collected post exposure and were reared to be infected (Figure

123 1). Offspring from the same parent and week $P E$ were housed together as a set for the duration of

124 the experiment. Two weeks following their collection, hatched offspring were moved to new jars

125 with $450 \mathrm{~mL}$ volume of well water. At ten weeks, 4-7 offspring from each set were randomly

126 selected to continue and moved to a jar of fresh well water. At twelve weeks, 4-6 offspring from

127 each set were individually exposed to 5 miracidia in well plates. Unexposed offspring were

128 discarded. The exposed offspring continued to be housed together for the duration of the

129 experiment with water changes occurring two times a week.

130 Infection was verified by cercariae shedding 4-6 weeks after parasite exposure.

131 Prevalence was recorded as the proportion of infected offspring in each set 6 weeks following

132 parasite exposure. Virulence was quantified by the cercariae output 6-10 weeks following

133 parasite exposure (Figure 1). Virulence was only quantified in the groups of offspring with a

134 minimum of 3 infected snails. If more than 3 offspring were infected, 3 infected individuals were

135 randomly chosen, the unselected offspring were discarded. The selected snails were placed

136 individually into well plates with 10 milliliters of well water, where they were exposed to light

137 for 1 hour to induce cercariae emergence. 333 microliter aliquots were taken from each of the 3 
138 wells to total a 999-microliter aliquot. Parasite virulence was calculated by averaging the total

139 number of cercariae collected from the three aliquots.

140 Utilizing the offspring prevalence data from 6 weeks following exposure, a two-

141 proportion $\mathrm{z}$ test was applied to test whether infection prevalence was significantly different in

142 offspring based on parental infection status. Virulence data was Box-Cox transformed to satisfy

143 the statistical assumptions for analysis. Our analysis showed that an exponent of 0.28 provided

144 the best fit to a normal distribution. Data were back transformed for use in figures. Virulence

145 data were analyzed using a general linear mixed model (GLMM) with parental treatment groups

146 as a fixed factor and parental ID and offspring weeks post exposure as random effects. When

147 analyzing virulence in the offspring associated with parental age, we used a GLMM with

148 parents' week post exposure as a fixed effect and parental ID, offspring week post exposure, and

149 parental infection status as random effects.

150 A significant increase in reproduction was observed in the infected parent group

151 compared to controls at three weeks following parasite exposure. A decrease in fecundity is

152 visible in the infected parent group as time progresses and the infection becomes patent (Figure

153 2).

154 Offspring produced by infected parents two weeks post exposure have a significantly

155 higher infection prevalence than offspring produced at the same timepoint from the control

156 parents $(\chi 2=16.906, \mathrm{p}<0.001)$. However, no other week PE exhibited a significant difference

157 in infection prevalence between offspring of infected and control parents (Week 4 PE $\chi 2=0.156$,

$158 \mathrm{p}=0.693$; Week 6 PE $\chi 2=0.059, \mathrm{p}=0.809$; Week 7 PE $\chi 2=0.547, \mathrm{p}=0.460)$. Parasite

159 virulence did not differ between infected offspring from infected parents or control parents for 
160 any time period post exposure (Week $4 \mathrm{PE} \mathrm{F}_{1,221}=1.315, \mathrm{p}=0.253$; week $6 \mathrm{PE} \mathrm{F} 1,124=2.659, \mathrm{p}$

$161=0.106 ;$ week $7 \mathrm{PE} \mathrm{F}_{1,72}=2.344, \mathrm{p}=0.130 ;$ Figure 3 )

162 Parental investment theory predicts that if organisms can recognize an environmental

163 challenge, they can make context-based investments into their offspring with non-genetic

164 resources (Rosengaus et al. 2017). In S. mansoni infections of B. glabrata, a decrease in

165 reproduction is obligate, as snails lose reproductive ability due to parasitic castration. There is

166 also a period after parasite exposure and before castration that infected snails can selectively

167 allocate resources to maximize their reproduction. Fecundity compensation represents a life

168 history strategy to maximize reproductive output. In this study, fecundity compensation was

169 observed in the third week following infection (Figure 1). However, accessing fecundity alone

170 does not forecast the adaptiveness of offspring to parasitic challenges (Stahlschmidt et al. 2013).

171 Offspring adaptiveness in a parasite environment can be estimated by their resistance to

172 parasitic infection. Evidence for an immune priming response in B. glabrata already challenges

173 the traditional assumption that invertebrates lack immune memory (Portela et al. 2013) by

174 indicating that resistance can be generated within generations. Furthermore, examples of

175 transgenerational immune priming in an array of invertebrates (Mitchell and Read 2005; Baron

176 et al. 2013; Rosengaus et al. 2017) are indicative that inherited invertebrate immunity can extend

177 beyond genetic inheritance. TGIP can occur via deliverance of active immune components to

178 offspring or by heightened endogenous immune function (Roth et al. 2018). TGIP in snails

179 parasitized with $S$. mansoni could suggest that $S$. mansoni resistance is carried to offspring by

180 processes beyond genetic changes. However, the results of this study do not provide support for

181 TGIP in this system. In contrast, offspring from infected parents 2 weeks PE exhibited a

182 significantly higher infection prevalence than offspring from uninfected parents. In fact, 
183 offspring from both infected and uninfected parents in week 2 demonstrated significantly lower

184 prevalence than offspring at all other time periods. This result suggests that increased parasite

185 resistance in offspring may be generated by healthier parents. Assessing infection virulence

186 could provide a stronger reflection of whether PE 2 offspring demonstrated increased resistance.

187 If the virulence of infections was lower in offspring from infected parents, this could indicate

188 that the hosts had a more resistant/tolerant relationship with the parasite. Unfortunately, we were

189 unable to assess virulence in week 2. The prevalence of infection was so low in the offspring

190 from the control parent PE 2 group, that the number of individuals in the samples did not meet

191 our standards to be analyzed for virulence. Virulence data were collected for offspring from

192 weeks 4, 6, and 7 PE.

193 There was no indication of increased resistance from offspring of infected parents at

194 weeks 4, 6, and 7 post exposure. Parental infection status did not confer any significant

195 difference in virulence or infection prevalence within those offspring groups (Figure 3).

196 Offspring from both the infected and control groups at the PE2 timepoint exhibited a

197 significantly lower prevalence than offspring from all other timepoints. Differences in the

198 prevalence of the PE 2 offspring may be a result of a healthier parent early in an infection. The

199 results of this study do not support TGIP even though immune priming has been observed in this

200 system (Portela et al. 2013). TGIP may not be an adaptive response in this host-parasite system.

201 TGIP only benefits offspring if it is predictive of challenges those offspring will experience

202 (Roth et al. 2018). The low prevalence of S. mansoni infections in natural snail populations,

203 (often much less than five percent), may make the cost of implementing TGIP exceed the

204 potential benefit (Minchella 1985). 
We acknowledge the members of the Minchella lab group for their assistance in

206 experimental set-up and data collection. We would also like to thank T.J. Vannatta who provided

207 valuable support in the statistical analysis. B. glabrata snails provided by the NIAID

208 Schistosomiasis Resource Center of the Biomedical Research Institute (Rockville, MD) through

209 NIH-NIAID Contract HHSN272201700014I for distribution through BEI Resources. O.J.

210 Lockyear was supported by a Cable-Silkman Undergraduate Fellowship. S.O Gutierrez was

211 supported by the National Science Foundation Graduate Research Fellowships Program.

212

213

214

215

216

217

218

219

220

221

222

223

224

225

226

227 
References:

Baron, Olga Lucia, Pieter van West, Benoit Industri, Michel Ponchet, Géraldine Dubreuil, Benjamin Gourbal, Jean-Marc Reichhart, and Christine Coustau. 2013. "Parental Transfer of the Antimicrobial Protein LBP/BPI Protects Biomphalaria Glabrata Eggs against Oomycete Infections (Parental Transfer of Immunity).” Edited by Matty Knight. PLoS Pathogens 9 (12): e1003792. https://doi.org/10.1371/journal.ppat.1003792.

Gleichsner, Alyssa M., Jessica A. Cleveland, and Dennis J. Minchella. 2016. “One StimulusTwo Responses: Host and Parasite Life-history Variation in Response to Environmental Maintenance of Schistosoma Mansoni, with Observations on Three Schistosome/Snail Host

Littlefair, Joanne E., Alice M. Laughton, and Robert J. Knell. 2017. “Maternal Pathogen

243 Minchella, Dennis J. 1985. "Host Life-History Variation in Response to Parasitism.” Article. Parasitology 90 (1): 205-16. https://doi.org/10.1017/S0031182000049143.

245 Minchella, Dennis J., and Philip T. Loverde. 1981. "A Cost of Increased Early Reproductive Effort in the Snail Biomphalaria Glabrata.” Article. The American Naturalist 118 (6): 876-

Mitchell, Suzanne E, and Andrew F Read. 2005. "Poor Maternal Environment Enhances 272 (1581): 2601. https://doi.org/10.1098/rspb.2005.3253. 
Nowak, Martin A., and Robert M. May. 1994. "Superinfection and the Evolution of Parasite Virulence.” Article. Proceedings of the Royal Society B: Biological Sciences 255 (1342): 81-89. https://doi.org/10.1098/rspb.1994.0012.

254 Pinaud, Silvain, Julien Portela, David Duval, Fanny C Nowacki, Marie-Aude Olive, JeanFrançois Allienne, Richard Galinier, et al. 2016. “A Shift from Cellular to Humoral Responses Contributes to Innate Immune Memory in the Vector Snail Biomphalaria Glabrata.” Article. PLoS Pathogens 12 (1): e1005361.

Portela, Julien, David Duval, Anne Rognon, Richard Galinier, Jérôme Boissier, Christine https://doi.org/10.1371/journal.ppat.1005361.

Rosengaus, Rebeca B., Nicole Hays, Colette Biro, James Kemos, Muizz Zaman, Joseph Murray, Coustau, Guillaume Mitta, André Théron, and Benjamin Gourbal. 2013. "Evidence for Specific Genotype-Dependent Immune Priming in the Lophotrochozoan Biomphalaria

Roth, Olivia, Anne Beemelmanns, Seth M Barribeau, and Ben M Sadd. 2018. "Recent Advances in Vertebrate and Invertebrate Transgenerational Immunity in the Light of Ecology and 
PLoS Neglected Tropical Diseases 10 (7): e0004794.

275 https://doi.org/10.1371/journal.pntd.0004794.

276 Stahlschmidt, Zachary R., Njal Rollinson, Madison Acker, and Shelley A. Adamo. 2013. “Are All Eggs Created Equal? Food Availability and the Fitness Trade-off between Reproduction

284 We would like to thank the members of the Minchella lab group for their assistance in data

285 collection and T.J. Vannatta who provided valuable support in the statistical analysis. O.J.

286 Lockyear was supported by a Cable-Silkman Undergraduate Fellowship. S.O Gutierrez was

287 supported by the National Science Foundation Graduate Research Fellowships Program. 


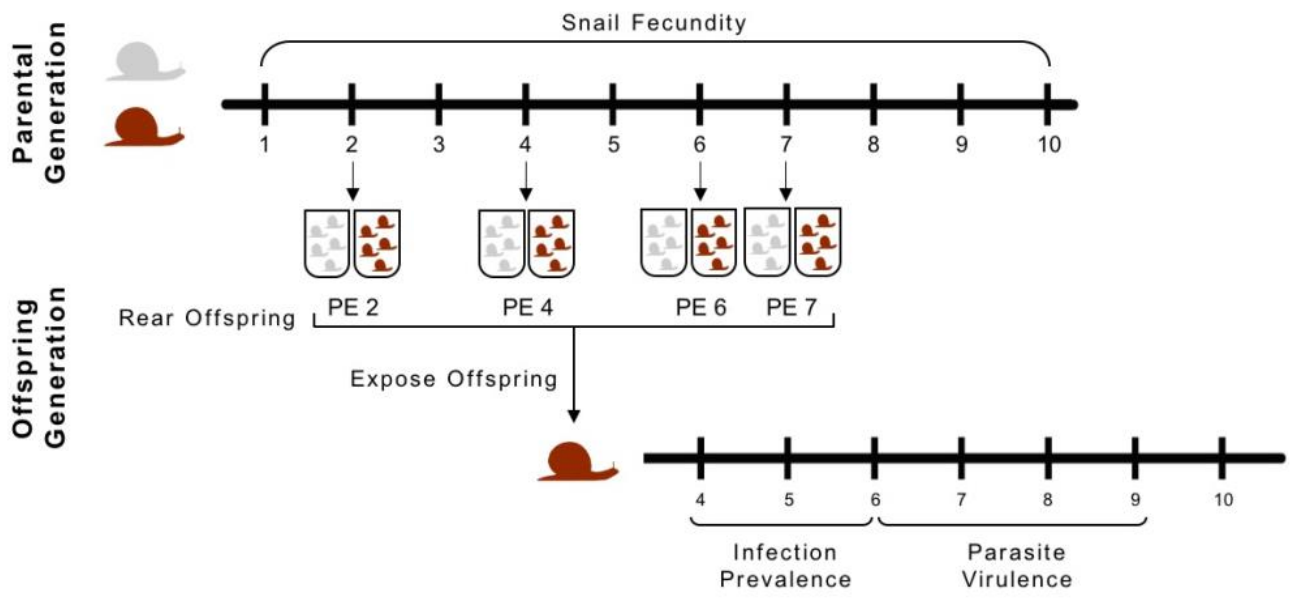

298 Figure 1. Experimental Design. Snail fecundity for infected (red) and uninfected control (gray)

299 parental generation was recorded for 10 weeks post exposure (PE). Offspring from weeks 2, 4, 6,

300 and 7 PE were collected and reared from both control and infected parents. The offspring

301 generation was then exposed to S. mansoni at 12 weeks. Infection prevalence (at weeks 4-6) and

302 parasite virulence (at weeks 6-9) were recorded for of the offspring generation.

303

304

305

306

307

308

309 


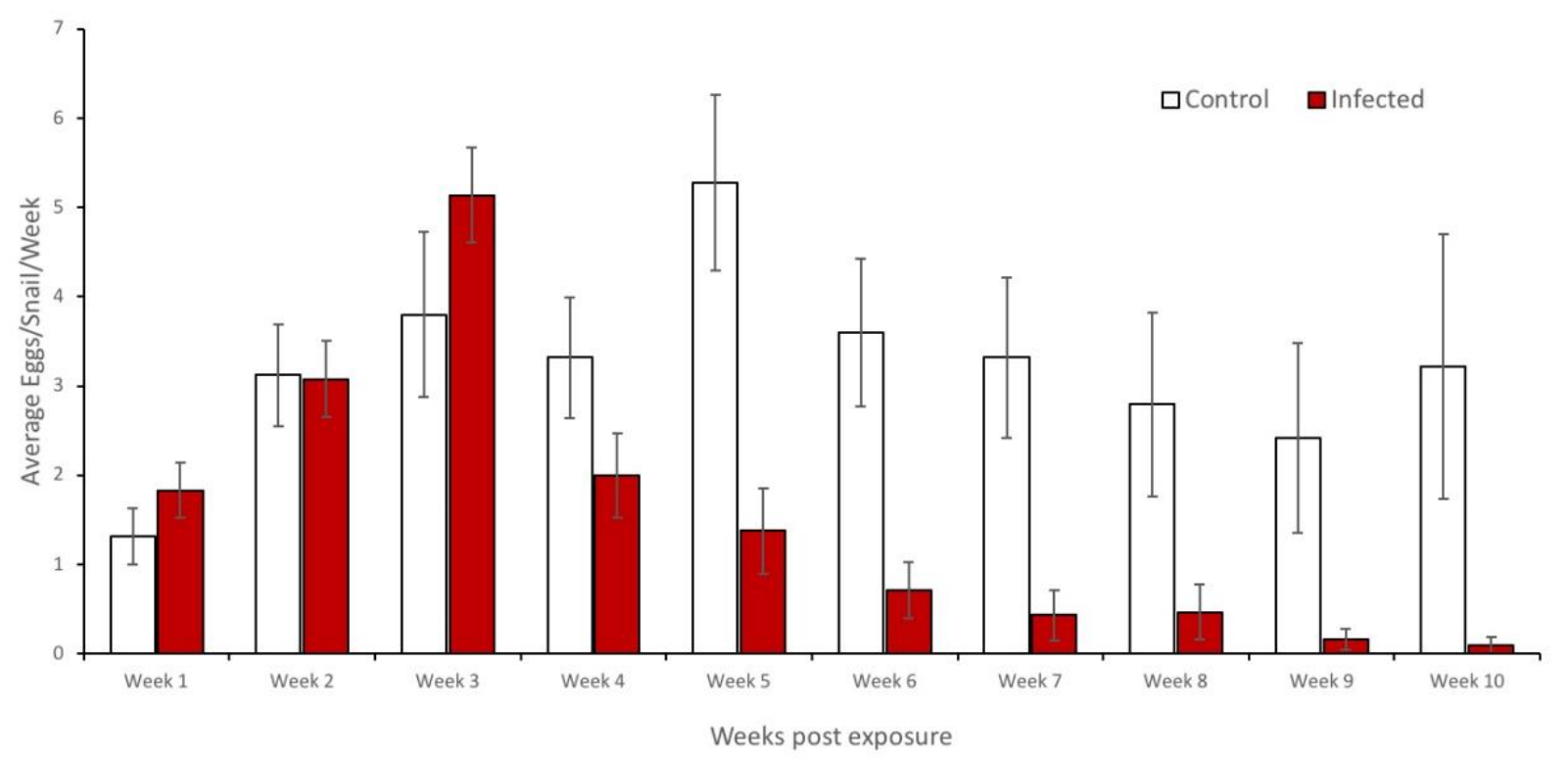

316 Figure 2. Snail fecundity of the parental generation over 10 weeks. Fecundity was calculated as

317 the average reproductive output (number of egg masses) from each snail per week. 


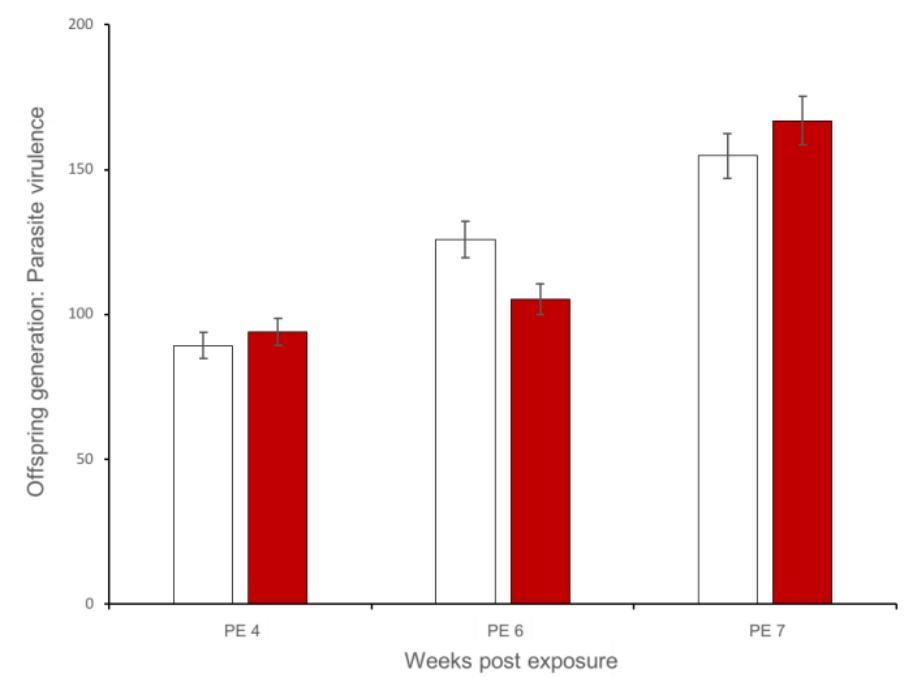

332 Figure 3: Parasite virulence (average number of cercariae) of offspring reared from parental

333 generation at weeks 4, 6, and 7 post exposure with relation to parent infection status (control vs.

334 infected). Virulence was not significantly different based on parental infection status for any

335 week post exposure $\left(\operatorname{PE} 4 \mathrm{~F}_{1,221}=1.315, \mathrm{p}=0.253\right.$; $\mathrm{PE} 6 \mathrm{~F}_{1,124}=2.659, \mathrm{p}=0.106 ; \mathrm{PE} 7 \mathrm{~F}_{1,72=}$ $3362.344, \mathrm{p}=0.130)$. 